\title{
Lymphatic Venous Anastomosis Can Release the Lymphedema-Associated Pain of Upper Limb after Breast Cancer Treatment
}

\author{
Makoto Mihara, MD ${ }^{1,2,3, *}$ Hisako Hara, MD ${ }^{1, *}$ Han Peng Zhou, MD ${ }^{4}$ Shuichi Tange, MD \\ Kazuki Kikuchi, $\mathrm{MD}^{3}$ Yoshihisa Kawakami, $\mathrm{MD}^{6}$
}

${ }^{1}$ Department of Lymphatic and Reconstructive Surgery, Saiseikai Kawaguchi General Hospital, Saitama, Japan

${ }^{2}$ Department of Plastic and Reconstructive Surgery, Avenue Cell Clinic, Tokyo, Japan

${ }^{3}$ Department of Plastic and Reconstructive Surgery, Nadogaya

Hospital, Chiba, Japan

${ }^{4}$ Department of Clinical Residency, Fujieda Municipal General

Hospital, Shizuoka, Japan

${ }^{5}$ Department of Clinical Residency, NTT Medical Center Tokyo,

Tokyo, Japan

${ }^{6}$ Department of Plastic and Reconstructive Surgery, Fukuoka

University, Fukuoka, Japan

J Reconstr Microsurg Open 2018;3:e1-e7.

\begin{abstract}
Address for correspondence Hisako Hara, MD, Department of Lymphatic and Reconstructive Surgery, Saiseikai Kawaguchi General Hospital, 5-11-5 Nishikawaguchi, Kawaguchi-Shi, Saitama 332-0021, Japan (e-mail: hisakohara.prs@gmail.com).
\end{abstract}

\begin{abstract}
Keywords

- lymphedema

- visual analog scale

- lymphaticovenous anastomosis

Background Sometimes, chronic pain in the arm or chest could occur in postmastectomy patients. Although the pathology of the pain is unclear, the involvement of neurological mechanism, cicatricial contracture, or lymphedema is considered. The purpose of this study was to investigate the effectiveness of lymphaticovenous anastomosis (LVA) in reducing chronic pain in upper limb lymphedema patients.

Patients and Methods This prospective study included consecutive 13 patients with upper limb lymphedema who received LVA. Preoperative lymphoscintigraphy and indocyanine green lymphography were performed. Pre- and postoperative pain scale were recorded using the visual analog scale (VAS). The number of cellulitis 1 year before and after LVA were compared. LVA was performed under local anesthesia, using a surgical microscope, and 12-0 nylon suture was used in the anastomosis.

Results Two out of 13 patients were excluded from this study, and 11 patients were subjected to this study. All subjects were females with an average age of 64.3 years. The average lymphedema duration was 76.7 months. The average number of LVA sites was 5.7 per limb and the average follow-up period was 10.6 months. The average pre- and postoperative VAS scores were 3.5 and 0.59 , respectively; the significant decrease was observed $(p=0.017)$. Three of the patients who had experienced cellulitis (once, twice, and four times, respectively) did not develop any cellulitis after LVA.

Conclusion LVA was shown to be an effective surgical remedy for treating the lymphedema-associated pain of upper limb after breast cancer treatment.
\end{abstract}

Makoto Mihara and Hisako Hara contributed equally to this work.

received

December 19, 2016 accepted after revision August 24, 2017
DOI https://doi.org/

10.1055/s-0037-1607306. ISSN 2377-0813.
Copyright $\odot 2018$ by Thieme Medical Publishers, Inc., 333 Seventh Avenue, New York, NY 10001, USA. Tel: +1(212) 584-4662.
License terms

(c) (1) $\ominus$ (\$) 
Studies have shown that there is an 8 to $56 \%$ chance of developing secondary lymphedema in patients who have undergone postmastectomy breast cancer treatments, such as axillary lymph node dissection and radiation therapy. ${ }^{1,2}$ On the other hand, chronic pain could also become a complication in these patients. The lymphedema-associated pain (LAP) refers to the chronic pain that occurs in the affected upper limb after mastectomy. ${ }^{3-5}$ LAP is often difficult to distinguish from the wound pain, and its etiology is thought to be a neurological cause. Current treatments for LAP include the use of analgesics and antidepressants; however, a new surgical therapy involving the transplantation of vascularized lymph node to the dissected region has also shown to be effective in reducing LAP. $^{6,7}$ Vascularized lymph node transfer not only prevents cicatricial contracture and nerve entrapment by resection of the damaged tissues, but it can also improve the lymphatic flow in the area by promoting lymphogenesis, ${ }^{8}$ hence relieving the lymphatic pressure in the area and reducing pain.

We perform lymphaticovenous anastomosis (LVA) on patients with postmastectomy secondary upper limb lymphedema. ${ }^{6-8}$ LVA is a low-invasive surgical treatment for lymphedema, which can be performed under local anesthesia and only involves incision up to the subcutaneous layer. ${ }^{9-12}$ Chang et al have previously reported the effectiveness of LVA in treating postmastectomy upper limb lymphedema in a prospective study. ${ }^{13}$ In this current study, we were able to reduce the degree of chronic pain in the affected limbs by performing LVA to patients with upper limb lymphedema. The purpose of this study was to investigate the effectiveness of LVA in reducing LAP in the upper limbs.

\section{Patients and Methods}

This prospective study included consecutive 13 patients with upper limb lymphedema who received LVA at Saiseikai Kawaguchi General Hospital, Saitama, between the period of August 2014 and October 2015. Exclusion criteria of the study included patients who were unable to be clinically followed up in our clinic postoperatively.

Each patient was preoperatively classified under the International Society of Lymphology Classification. ${ }^{14}$ Preoperative lymphoscintigraphy and indocyanine green (ICG) lymphography were performed to diagnose and classify each case into appropriate stages of lymphedema. ICG lymphography was performed by 0.1 to $0.2 \mathrm{~mL}$ subcutaneous injection of ICG dye (0.25\% diagnogreen, Daiichi Sankyo Company Ltd., Tokyo, Japan) in the second interdigital space. Photodynamic eye system (PDE, Hamamatsu Photonics K.K., Hamamatsu, Japan) was then used to visualize the injected dye. Each case was classified using the dermal backflow staging classification. ${ }^{15,16}$ Lymphoscintigraphy was performed by injecting $0.3 \mathrm{~mL}$ (222 MBq) of 99mTc-labeled albumin radioisotope dye subcutaneously into the second interdigital space bilaterally. Scintigram images were taken $15,30,60$, and 90 minutes after the injection. Each case was then classified using the Maegawa classification. ${ }^{17}$

Pre- and postoperative pain scale of each case were recorded using the visual analog scale (VAS). The frequency of cellulitis 1 year before and after LVA were compared for patients who have been clinically followed up for over 12 months postoperatively. Cellulitis was defined by the redness and pyrexia of the affected limb. The stiffness of the skin and subcutaneous tissue was evaluated based on the descriptions in the patients' medical chart. Preoperative upper limb circumferences were measured at six predefined sites of the affected limb: $15 \mathrm{~cm}$ proximal from the elbow joint, $10 \mathrm{~cm}$ proximal from the elbow joint, at the elbow joint, $5 \mathrm{~cm}$ distal from the elbow joint, at the wrist, and at the dorsum of the hand. In one patient, only two out of the six predefined measurements were taken preoperatively; hence, only the postoperative measurements from the same two sites were taken into evaluation. The postoperative change of the limb circumference was calculated using the following equation:

\section{Change rate $=\left(\sum\right.$ preoperative circumferences $-\sum$ postopera- tive circumferences)/ $\sum$ preoperative circumference}

LVA was performed under local anesthesia. Lymphatic vessels suitable for anastomosis were marked using ICG lymphography, and veins were marked using a noninvasive infrared camera. LVA was performed at sites where the lymphatic vessel approaches the marked veins. ${ }^{9,10}$ The surgery was performed under a surgical microscope, and the anastomosis was performed using 12-0 nylon suture. We selected the lymphatic vessels of ectasis type if possible because it is thought to lead to the best result. ${ }^{18,19}$ However, if the lymphatic vessels of ectasis type did not found, we anastomosed other types.

Essentially the same compression therapy was performed before and after the surgery; however, the frequency of wearing compression therapy was reduced for patients who showed significant postoperative improvement of edema. Pre- and postoperative frequency of wearing elastic sleeves was recorded.

This study was approved by the ethical committee at our hospital and written informed consents were collected from all participating patients.

\section{Results}

Two out of the consecutive 13 upper limb secondary lymphedema patients who received LVA treatment at the Saiseikai Kawaguchi General Hospital, Saitama, between the periods of August 2014 and October 2015 were excluded from this study as they did not have any postoperative follow-up at our clinic. One patient had kidney failure during the postoperative observational period and developed anasarca; hence, we had to evaluate her upper limb lymphedema condition from her last postoperative visit before the exacerbation of her renal failure. Eventually, 11 patients were subjected to this study ( - Table $\mathbf{1}$ ). All patients were female with an average age of 64.3 years (range: $40-84$ years). The average body mass index of the subjects was $24.6 \mathrm{~kg} / \mathrm{m}^{2}$ (range: $19.8-33.0 \mathrm{~kg} / \mathrm{m}^{2}$ ). The etiology of lymphedema was breast cancer treated by mastectomy and axillary lymph node dissection for all subjects. Nine subjects underwent postoperative radiation therapy. Seven subjects had right upper limb lymphedema, and four subjects had left 
Table 1 Patient summary

\begin{tabular}{|c|c|c|}
\hline Characteristics & & Value \\
\hline Number of patient & & 11 \\
\hline \multirow[t]{2}{*}{ Age, y } & Average & 65.4 \\
\hline & Range & $40-84$ \\
\hline \multirow[t]{2}{*}{ Sex } & Female & 11 \\
\hline & Male & 0 \\
\hline \multirow[t]{2}{*}{ Follow-up, mo } & Average & 10.6 \\
\hline & Range & $2.5-19$ \\
\hline Causative disease & Breast cancer & 11 \\
\hline \multirow[t]{2}{*}{ Lymphadenectomy } & Positive & 11 \\
\hline & Negative & 0 \\
\hline \multirow[t]{2}{*}{ Radiotherapy } & Positive & 9 \\
\hline & Negative & 2 \\
\hline \multirow[t]{2}{*}{ History of cellulitis } & Positive & 3 \\
\hline & Negative & 8 \\
\hline \multirow[t]{4}{*}{ ISL classification } & 1 & 0 \\
\hline & $2 a$ & 0 \\
\hline & $2 b$ & 9 \\
\hline & 3 & 2 \\
\hline
\end{tabular}

Abbreviation: ISL, International Society of Lymphology.

upper limb lymphedema. Nine subjects were classified as stage $2 \mathrm{~b}$ and two subjects as stage 3 by the International Society of Lymphology classification. The average lymphedema duration among the patients was 76.7 months (range: $16-226$ months), and all patients were subjected to compression therapy using compressional sleeves.

Three of the operations were performed by two operators (M.M. and H.H.) simultaneously using two surgical microscopes, while the others were performed by one operator (M.M.). The average number of LVA sites was 5.7 (range: 5-8 sites) per limb and the average operation time was $159.2 \mathrm{~min}-$ utes (range: 122-230 minutes). The average postoperative follow-up period was 10.6 months (range: $2.5-19$ months).

The changes in the pre- and postoperative VAS scores are shown in -Fig. 1. The VAS scores had a tendency to be decreased postoperatively. The average change rate of upper limb circumference was $-1.43 \%$ (range: -9.9 to $4.63 \%$, $p=0.86$ ). The average pre- and postoperative VAS scores were 3.5 (range: $0-8$ ) and 0.59 (range: $0-3$ ) respectively; hence, a significant decrease in the postoperative VAS score was observed ( $p=0.017$ ).

Five of the patients wore elastic sleeves $(20 \mathrm{~mm} \mathrm{Hg})$ preand postoperatively. Two did not wear them at all. Three wore elastic sleeves $(20 \mathrm{~mm} \mathrm{Hg}$ ) preoperatively, but stopped wearing them postoperatively in two and decreased to thrice a week in one. One patient wore elastic sleeve thrice a week preoperatively because she did not like it, but wore it every day to be motivated by undergoing LVA. Five patients (45.5\%) could control lymphedema without wearing elastic sleeves every day postoperatively.

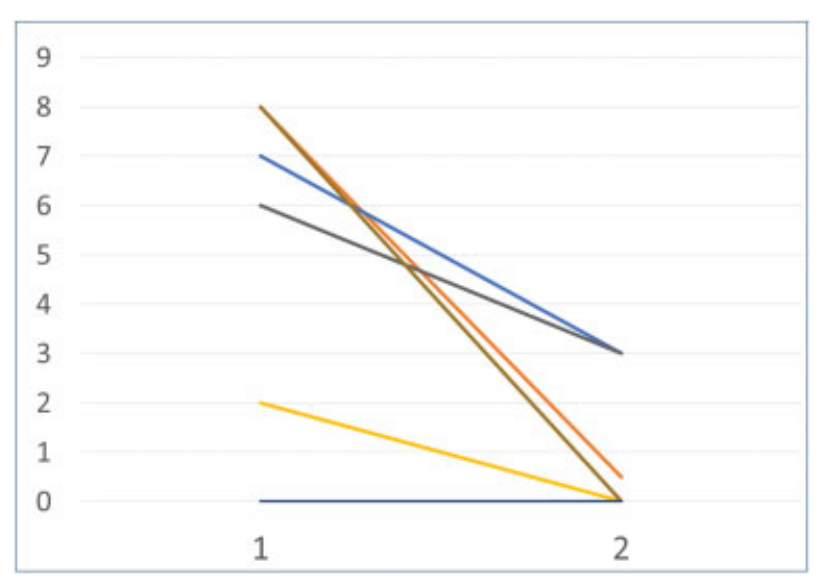

Fig. 1 Pre and postoperative pain scores using the visual analog scale.

The frequency of cellulitis was evaluated in six patients whose follow-up period was more than 12 months. Three of the subjects who had developed cellulitis in their affected limbs within 1 year before the surgery (once, twice, and four times, respectively) did not develop any cellulitis after the procedure.

\section{Case 1}

A 40-year-old patient diagnosed with stage 4 left breast cancer at the age of 38 years who was treated by total mastectomy and axillary lymph node dissection. Postoperative chemoradiation therapy by the taxane-based carcinostatic agent was introduced. Left upper limb lymphedema appeared 1 year after the cancer treatment and LAP developed on her entire left upper limb, precordium, and back. The pain on her left limb, as well as her left shoulder and precordium, was rated 8 on the VAS score ( - Fig. 2A). Furthermore, the patient developed lymphorrhea from infected cutaneous ulcers in her axilla and required dressing change several times a day for over 2 months. The patient was first referred to another hospital for conservative therapy; however, she was unable to continue manual lymph drainage and compression therapies due to severe pain and was referred to our hospital for surgical solutions.

Lymphoscintigraphy (-Fig. 3) and ICG lymphography ( - Fig. 4, - Video 1) confirmed that the sites of dermal backflow pattern matched the sites where the chronic pain was felt; thus, implying that lymph congestion may be the cause of pain. Several functional collecting lymphatic vessels suitable for LVA were identified in the forearm and brachium of the patient 15 minutes after the ICG dye was injected subcutaneously.

\section{Video 1}

Case 1: The preoperative ICG lymphographic imaging. Dermal backflow can be observed from the left arm expanding toward the shoulder and the precordium. ICG, indocyanine green. Online content including video sequences viewable at: www.thieme-connect. com/ejournals/html/doi/10.1055/s-0037-1607306. 

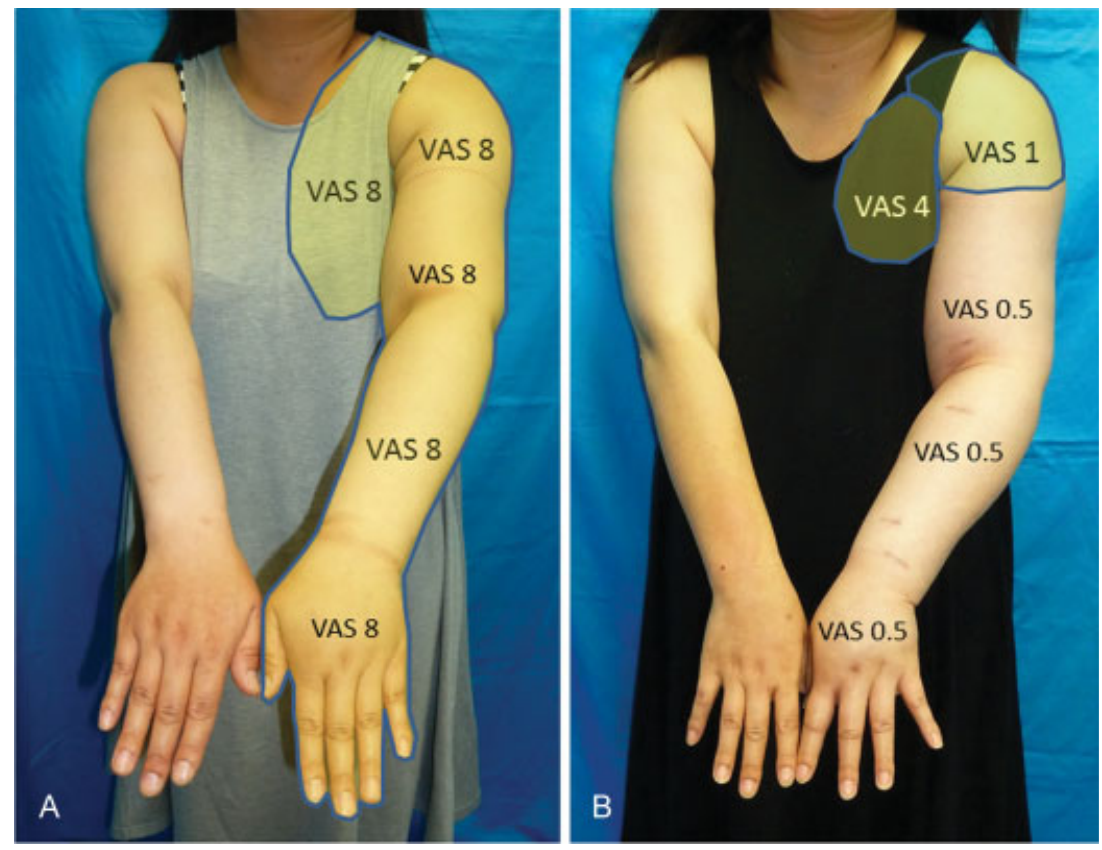

Fig. 2 Case 1: (A) Preoperative VAS scores of the patient. The Severe chronic pain was felt in the precordium expanding to the shoulder and the entire upper limb. (B) The postoperative VAS scores of the patient. Although the pain in the precordium remains due to cancer relapse and radiation therapy, pain level has decreased significantly in most areas. VAS, visual analog scale.

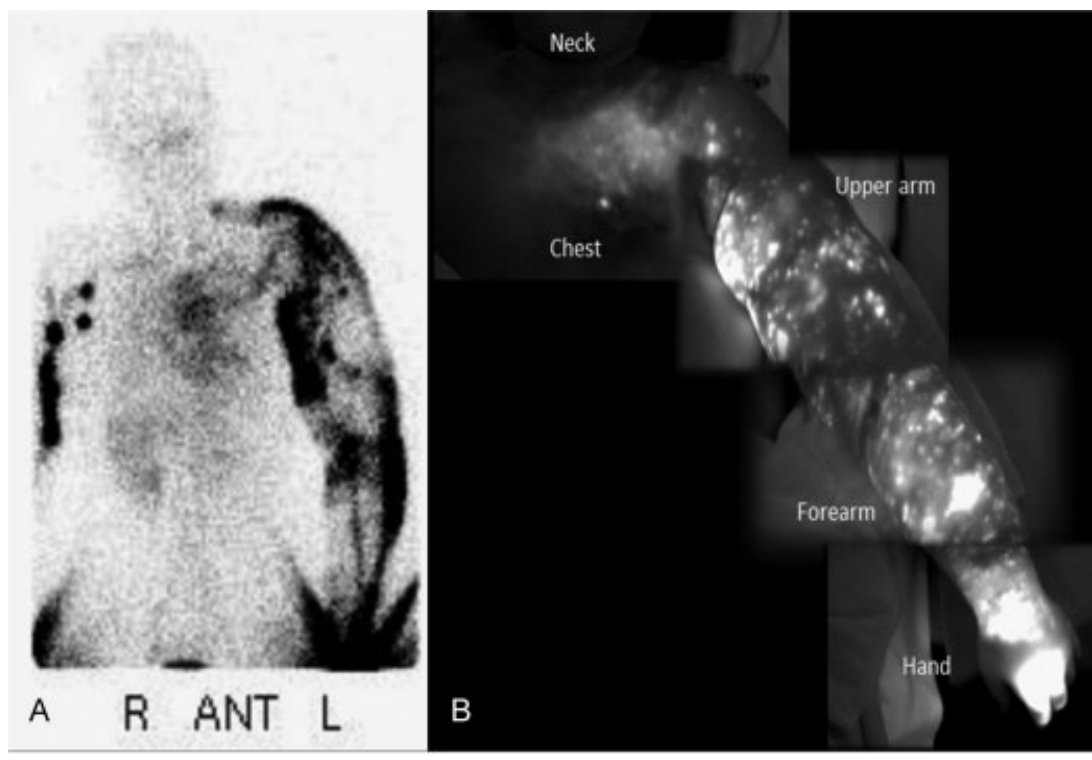

Fig. 3 Case 1: (A) Preoperative lymphoscintigraphic image 90 minutes after subcutaneous injection of radioisotope in the bilateral second interdigital space. Several collecting lymphatic ducts can be observed from the left forearm extending toward the brachium; however, dermal backflow can be observed in the left shoulder, medial margin of the left brachium, the left cubital fossa, and the lateral margin of the left forearm. (B) Preoperative ICG lymphographic image 30 minutes after subcutaneous injection of ICG dye in the second interdigital space. Dermal backflow can be observed in the entire left upper arm, the left shoulder, and the precordium. ANT, anterior; ICG, indocyanine green; POST, posterior; R, right; L, left.

LVA was performed under local anesthesia. A total of five sites (two at the wrist, one at the lateral margin of the forearm, two at the brachium) were selected for the procedure (-Fig. 5). Sclerosis type lymphatic vessels were identified in the brachium. ${ }^{19}$ Dilated and relatively functional lymphatic vessels were identified at the forearm and the wrist. For these well-functioning vessels, we were able to observe the lymph fluid flowing into the vein after the anastomosis was constructed (-Fig. 5 [bottom left]). The total operating time was 3 hours and 22 minutes.

Upper limb edema decreased after 2 weeks, and pain gradually decreased 3 months after the procedure with a 


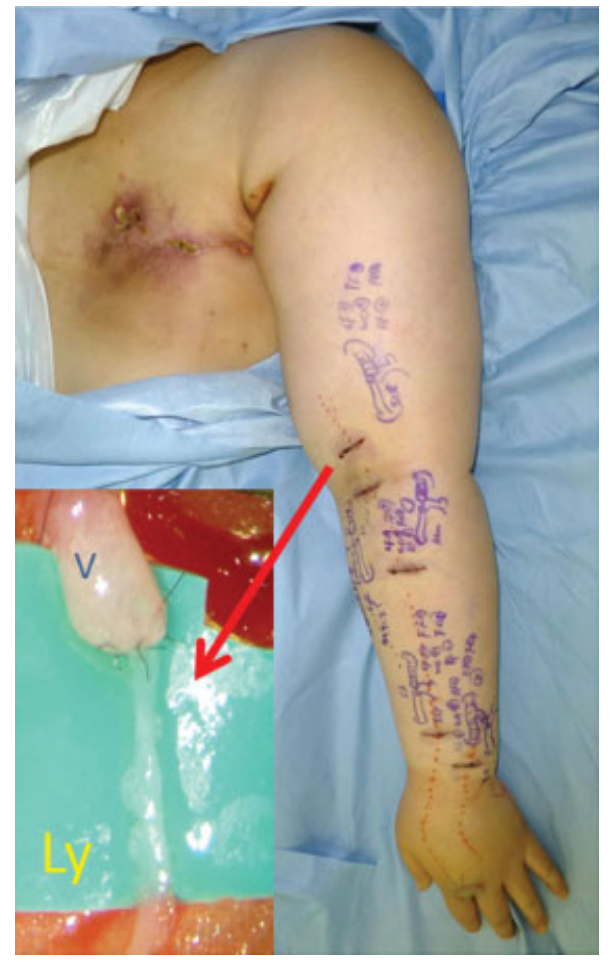

Fig. 4 Intraoperative image of LVA. LVA was performed at five predefined sites under local anesthesia. The lymphatic vessels identified in the medial margin of the forearm were all fragile with a diameter of approximately $0.2 \mathrm{~mm}$. LVA, lymphaticovenous anastomosis.

final VAS score of 1 in the shoulder and 0.5 in the whole upper limb region (-Figs. 1 and $\mathbf{2}$ ). The pain in the precordial region decreased to a VAS score of 4 . Tissue stiffness of the arm improved slightly; however, the limb circumference failed to show any significant improvement postoperatively. Lymphorrhea and the malodor from the axillary ulcers have also significantly decreased after the procedure, and the frequency of dressing change required decreased to once a day with the use of adhesive plasters only.

\section{Case 2}

A 69-year-old female patient diagnosed with right breast cancer at the age of 58 years who had been treated by total mastectomy with axillary lymph node dissection followed by radiation therapy. Right upper limb lymphedema appeared 7 years after the surgery and gradually exacerbated. LAP also slowly manifested in the affected limb in addition to 12 or more occurrences of cellulitis per year. The patient underwent LVA surgery 3 years ago, and the number of annual cellulitis occurrences had been reduced to four times per year. The chronic pain scored 7 on the VAS. Lymphoscintigraphy and ICG lymphography revealed expanding dermal backflow patterns in the right upper arm spreading toward the forearm.

The LVA procedure was performed under local anesthesia at four predefined sites in the affected limb: two sites at the wrist, one at the medial margin of the forearm, and one at the cubital fossa. A total of six anastomoses were made among these sites. The total operating time was 2 hours and 9 minutes. The surgery was completed without any major postoperative complications.

Although the postoperative change rate of the right upper limb circumference exacerbated by $20.9 \%$, the VAS score improved from 7 to 0 postoperatively and there was no occurrence of cellulitis during the 15-month follow-up period. The patient continued the use of compression sleeves after the procedure.

\section{Discussion}

By introducing LVA to patients with postmastectomy upper limb lymphedema, we were able to reduce the degree of LAP. Upper limb lymphedema often develops in patients who have
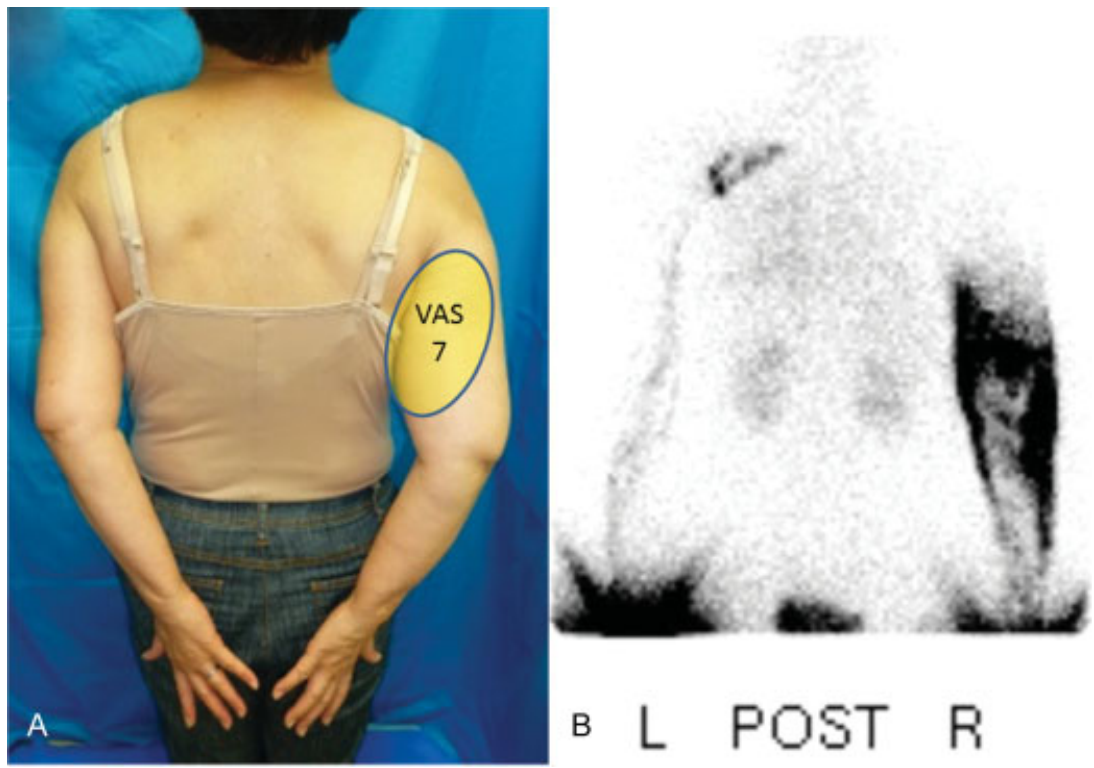

Fig. 5 Back shot of case 2: (A) Preoperative VAS scores of the patient. Severe chronic pain (VAS: 7) was felt in the right brachium region. (B) Preoperative lymphoscintigraphic image 90 minutes after subcutaneous injection of radioisotope in the bilateral second interdigital space. Dermal backflow can be observed in the right brachium and elbow region. ANT, anterior; ICG, indocyanine green; POST, posterior; R, right; L, left; VAS, visual analog scale. 
undergone breast cancer surgery and LAP may manifest in these patients. The etiology of the chronic pain caused by LAP is believed to derive from a neurological origin. Conventional treatments for LAP include the use of analgesics and antidepressants; however, medication therapy alone is not sufficient to control the pain caused by LAP. ${ }^{3-5}$ In this current study, by applying imaging techniques, such as lymphoscintigraphy and ICG lymphography, we were able to confirm that the site of lymphatic fluid congestion matched the sites where the chronic pain was felt by the patient; hence, we believe that there is a strong correlation between them. Also, cicatricial contracture of the tissues in the axilla may be a primary cause of chronic pain. In this manner, by understanding the etiology of the chronic pain in lymphedema patients, we will be able to choose the most appropriate treatment method for each patient. For instance, if the underlying cause of chronic pain was due to impaired lymphatic function, indicated by lymphoscintigraphy or ICG lymphography, then treatment for lymphedema should be performed to relieve the symptoms. ${ }^{6,7}$ On the other hand, if the cause of chronic pain was due to nerve compression or cicatricial contracture, then neurolysis or cicatrectomy followed by tissue flap transplantation should be the choice of treatment to relieve the pain.

Furthermore, the taxane-based regime was used for chemotherapy in some of the subjects included in this current study. Previous reports have shown that taxane-based agents are known to have an inhibitory effect on the systemic lymphatic function in patients. ${ }^{20,21}$ This was also confirmed in our study as a greater number of sclerosis type lymphatic vessels were observed in subjects who received taxane-based agents. ${ }^{9,10}$ Other surgical options for lymphedema, such as lymph node transfer, are not suitable for such patients whose lymphatic function is suppressed as it will lead to a greater risk of developing donor-site lymphedema. ${ }^{22}$ Hence, we believe that LVA would be the most appropriate surgical option for these patients. ${ }^{9-13}$

LVA is a minimally invasive surgery that can be performed under local anesthesia and only requires small incisions from 1 to $3 \mathrm{~cm}$. Due to its minimum invasiveness, it is possible to perform LVA on patients who are currently going through cancer treatment or in terminal patients as a part of palliative care. However, the possibility of cancer metastasis via the lymph-vein anastomosis must be taken into account; hence, a thorough explanation and informed consent must be obtained from the patient. ${ }^{23}$

In the present study, we did not observe a significant decrease in the limb circumference, although there was an average circumference change of $-1.4 \%$. We believe that the absence of circumference reduction does not equate to treatment failure because it is known that fibrotic tissue and fat tissue increased in lymphedema-affected extremities. $^{24,25}$ Therefore, in some cases, patients' symptom get better after LVA with the lymphatic drainage improvement, even if circumference reduction is small. The relief from the pain in the extremities should be a benefit for the lymphedema patients, even if the limb circumference is unchanged.

The limitation of this study was that only 13 subjects were included in the study; hence, a greater number of cases must be assessed in future studies to establish a higher level of clinical evidence.

In conclusion, LVA was shown to be an effective surgical remedy for treating LAP in upper limb lymphedema patients, and it also improved the annual frequency of cellulitis in the affected limb.

\section{Acknowledgment}

The authors thank the support of Isao Koshima (The University of Tokyo), Eri Saeki, Izumi Masuda, Hiroshi Minezaki, and Shigeru Harasawa (Saiseikai Kawaguchi General Hospital), without which this study could not have been completed.

\section{References}

1 Kilbreath SL, Refshauge KM, Beith JM, et al. Risk factors for lymphoedema in women with breast cancer: A large prospective cohort. Breast 2016;28:29-36

2 Paskett ED, Naughton MJ, McCoy TP, Case LD, Abbott JM. The epidemiology of arm and hand swelling in premenopausal breast cancer survivors. Cancer Epidemiol Biomarkers Prev 2007;16 (04):775-782

3 Vilholm OJ, Cold S, Rasmussen L, Sindrup SH. The postmastectomy pain syndrome: an epidemiological study on the prevalence of chronic pain after surgery for breast cancer. Br J Cancer 2008;99 (04):604-610

4 Kwekkeboom K. Postmastectomy pain syndromes. Cancer Nurs 1996;19(01):37-43 Review

5 Ganel A, Engel J, Sela M, Brooks M. Nerve entrapments associated with postmastectomy lymphedema. Cancer 1979;44(06): 2254-2259

6 Becker C, Assouad J, Riquet M, Hidden G. Postmastectomy lymphedema: long-term results following microsurgical lymph node transplantation. Ann Surg 2006;243(03):313-315

7 Becker C, Pham DN, Assouad J, Badia A, Foucault C, Riquet M. Postmastectomy neuropathic pain: results of microsurgical lymph nodes transplantation. Breast 2008;17(05):472-476

8 Mihara M, Iida T, Hara H, et al. Autologus groin lymph node transfer for "sentinel lymph network" reconstruction after headand-neck cancer resection and neck lymph node dissection: a case report. Microsurgery 2012;32(02):153-157

9 Mihara M, Hara H, Hayashi Y, et al. Upper-limb lymphedema treated aesthetically with lymphaticovenous anastomosis using indocyanine green lymphography and noncontact vein visualization. J Reconstr Microsurg 2012;28(05):327-332

10 Mihara M, Hara H, Furniss D, et al. Lymphaticovenular anastomosis to prevent cellulitis associated with lymphoedema. $\mathrm{Br} \mathrm{J}$ Surg 2014;101(11):1391-1396

11 Hara H, Mihara M, Seki Y, Todokoro T, Iida T, Koshima I. Comparison of indocyanine green lymphographic findings with the conditions of collecting lymphatic vessels of limbs in patients with lymphedema. Plast Reconstr Surg 2013;132(06):1612-1618

12 Boccardo F, Casabona F, De Cian F, et al. Lymphedema microsurgical preventive healing approach: a new technique for primary prevention of arm lymphedema after mastectomy. Ann Surg Oncol 2009;16(03):703-708

13 Chang DW, Suami H, Skoracki R. A prospective analysis of 100 consecutive lymphovenous bypass cases for treatment of extremity lymphedema. Plast Reconstr Surg 2013;132(05):1305-1314

14 International Society of Lymphology. The diagnosis and treatment of peripheral lymphedema: 2013 Consensus Document of the International Society of Lymphology. Lymphology 2013;46 (01):1-11 
15 Yamamoto T, Yamamoto N, Doi K, et al. Indocyanine greenenhanced lymphography for upper extremity lymphedema: a novel severity staging system using dermal backflow patterns. Plast Reconstr Surg 2011;128(04):941-947

16 Mihara M, Hara H, Araki J, et al. Indocyanine green (ICG) lymphography is superior to lymphoscintigraphy for diagnostic imaging of early lymphedema of the upper limbs. PLoS One 2012;7(06): e38182

17 Maegawa J, Mikami T, Yamamoto Y, Satake T, Kobayashi S. Types of lymphoscintigraphy and indications for lymphaticovenous anastomosis. Microsurgery 2010;30(06):437-442

18 Mihara M, Hara H, Tange S, et al. Multi-site lymphaticovenular bypass using supermicrosurgery technique for Lymphedema Management in lower lymphedema cases. Plast Reconstr Surg 2016;138(01):262-272

19 Mihara M, Hara H, Hayashi Y, et al. Pathological steps of cancerrelated lymphedema: histological changes in the collecting lymphatic vessels after lymphadenectomy. PLoS One 2012;7(07): e41126

20 Swaroop MN, Ferguson CM, Horick NK, et al. Impact of adjuvant taxane-based chemotherapy on development of breast cancer- related lymphedema: results from a large prospective cohort. Breast Cancer Res Treat 2015;151(02):393-403

21 Lee MJ, Beith J, Ward L, Kilbreath S. Lymphedema following taxane-based chemotherapy in women with early breast cancer. Lymphat Res Biol 2014;12(04):282-288

22 Pons G, Masia J, Loschi P, Nardulli ML, Duch J. A case of donor-site lymphoedema after lymph node-superficial circumflex iliac artery perforator flap transfer. J Plast Reconstr Aesthet Surg 2014;67 (01):119-123

23 Shimizu H, Maegawa J, Ho T, Yamamoto Y, Mikami T, Nagahama K. Cutaneous metastasis of pancreatic carcinoma as an initial symptom in the lower extremity with obstructive lymphedema treated by physiotherapy and lymphaticovenous shunt: a case report, review, and pathophysiological implications. Lymphology 2010; 43(01):19-24

24 Brorson H. Liposuction in Lymphedema Treatment. J Reconstr Microsurg 2016;32(01):56-65

25 Brorson H, Ohlin K, Olsson G, Nilsson M. Adipose tissue dominates chronic arm lymphedema following breast cancer: an analysis using volume rendered CT images. Lymphat Res Biol 2006;4(04): 199-210 